ASTHMA

\title{
Use of inhaled corticosteroids following discharge from an emergency department for an acute exacerbation of asthma
}

\author{
L Blais, M-F Beauchesne
}

Thorax 2004;59:943-947. doi: 10.1136/thx.2004.022475

See end of article for authors' affiliations .....................

Correspondence to: Professor L Blais, Université de Montréal, Faculté de Pharmacie, C P 6128, Montréal Québec, Canada H3C 3J7; lucie. blais@umontreal.ca

Received 28 January 2004 Accepted 27 August 2004

\begin{abstract}
Background: Most patients who have an asthma exacerbation leading to a visit to an emergency department (ED) will benefit from treatment with inhaled corticosteroids (ICS) at discharge. We investigated whether asthmatic children and adolescents were receiving ICS after discharge from the ED and identified the characteristics of patients and physicians associated with their use.

Methods: A cohort of 4042 asthmatic patients aged 5-17 years was selected from the administrative database of the Régie de l'assurance maladie du Québec between 1997 and 1999. The proportion of patients using ICS 1, 3, and 6 months after ED discharge was estimated. Using GEE models the independent contribution of sociodemographic variables, markers of asthma severity, prior use of healthcare services and ICS, and physician characteristics was investigated on the likelihood of using ICS after ED discharge.

Results: $68 \%$ of children and $51 \%$ of adolescents had a valid prescription for ICS in the month following discharge. At 6 months after discharge the corresponding figures were $77 \%$ and $60 \%$. The strongest predictors of ICS use were age, with adolescents being less likely to use ICS than children (OR 0.49; $95 \%$ $\mathrm{Cl} 0.43$ to 0.56 ), prior use of ICS (OR 2.28; $95 \% \mathrm{Cl} 2.00$ to 2.61 ), and filling a prescription for oral corticosteroids in the month following discharge (OR 2.29; $95 \% \mathrm{Cl} 2.03$ to 2.58). However, patients who had an ED visit or a hospital admission for asthma during the 6 months before discharge were not more likely to use ICS after discharge.

Conclusion: A large proportion of patients with clear markers of uncontrolled or severe asthma did not have a valid prescription for an ICS after discharge from the ED.
\end{abstract}

nhaled corticosteroids (ICS) are recognised as the most effective long term control medication for most patients with persistent asthma. ${ }^{1-3}$ Most patients who have an asthma exacerbation leading to visit to an emergency department (ED) will benefit from ICS treatment at discharge. The Canadian asthma guidelines for the treatment of asthma clearly recommend the prescription of ICS at discharge for all patients visiting an ED for an asthma exacerbation. ${ }^{1}$ Moreover, use of ICS after ED discharge has been shown to reduce the rate of relapse by $45-48 \%$ in two studies. ${ }^{45}$

Several studies have investigated the appropriateness of asthma management in the $\mathrm{ED},{ }^{6-14}$ but there is a paucity of literature on the outpatient management and, more specifically, the use of ICS after discharge from the ED. The three studies that have investigated the use of ICS after ED discharge identified in the literature reported a low rate of use. One small Canadian study based on 130 adult patients found that $25 \%$ of patients were discharged without a prescription for inhaled or oral corticosteroids. ${ }^{7}$ Another Canadian study reported that $50.9 \%$ of the 1293 patients under study (5-60 years of age) did not receive any ICS after ED discharge over a period that could be as long as 2 years. ${ }^{5}$ Singh and colleges also found a low rate of use of ICS among 119 patients discharged from the ED of the Massachusetts General Hospital in the USA, the rate being as low as $11 \%$ for patients without a primary care physician. ${ }^{15}$ However, to our knowledge no study has specifically investigated the use of ICS after discharge from an ED in children and adolescents with asthma.

We therefore conducted a population based study to estimate the proportion of children and adolescents with asthma who use an ICS after discharge from a visit to the ED.
We also investigated the patient and physician characteristics likely to predict the use of ICS after discharge.

\section{METHODS \\ Source of data}

Using the administrative database of the Régie de l'assurance maladie du Québec (RAMQ), the government body responsible for the administration of health services (including the provincial drug plan) in the Canadian province of Quebec, we recruited a cohort of asthmatic patients aged 5-17 years.

In Quebec all residents are covered by the RAMQ for the medical services but only approximately $50 \%$ of the population is covered by the RAMQ drug plan. The other half of the population is covered by private or group drug insurance plans provided at work. Among residents covered by the RAMQ drug plan at the time of the study (1998), 697127 were receiving social assistance and 1510485 aged 64 years or less and 872211 elderly subjects (aged $\geqslant 65$ years) were not receiving social assistance. ${ }^{16}$ In 1998 more than 390000 children in this age group were completely covered by the RAMQ and thus had free access to prescribed medications and medical services. ${ }^{16}$ Of those, 140000 were living in families receiving social assistance. The other 250000 children were living in families that had no access to a group insurance for their medications at work and were likely to represent a middle class population in terms of economic status. Families with the highest income are less likely to be insured by the RAMQ drug plan and are thus less likely to be included in this study.

The administrative database of the RAMQ contains information on prescriptions filled at pharmacies and on medical services dispensed at hospitals, EDs, and medical clinics. Pharmacy records include the unique patient identifier 
(encrypted), the name, dose, form and quantity of the medication dispensed, the duration and the date the prescription is filled, and the unique identifying number (encrypted) and specialty of the prescribing physician. Medical records include the unique patient identifier (encrypted), the date the service was dispensed, where it was dispensed (hospital, emergency department or medical clinic), the diagnosis, and the unique identifying number (encrypted) and specialty of the treating physician. RAMQ databases have been used extensively for epidemiological studies $^{17-21}$ and the information related to prescribed medications has been proved to be both valid and comprehensive. ${ }^{22}$

\section{Cohort of asthmatic patients visiting an ED}

From the pharmacy database of the RAMQ we first identified 32897 asthmatic patients aged 5-17 years. To be selected, patients were required to have filled at least two prescriptions for a short acting $\beta_{2}$ agonist (albuterol, fenoterol, epinephrine, orciprenaline, pirbuterol, or terbutaline) on or after their fifth birthday between 1 January 1997 and 31 December 1999. Patients using non-steroidal inhaled anti-inflammatory agents (disodium cromoglycate, nedocromil sodium and ketotifen) and leukotriene receptor antagonists (zafirlukast and montelukast) were excluded from the cohort because the frequency of use of these medications was less than 3\%. This cohort is thus a representative sample of patients covered by the RAMQ drug plan who visited the ED at the time of the study. There are 92 ED facilities in the province of Québec, 10 of which are at academic centres. The four paediatric only ED facilities in the province are in academic centres.

Patients entered the cohort on the date of the first ED visit for asthma (ICD-9 codes: 493.0, 493.1, 493.9) on or after 1 July 1997. For each patient the number of ED visits made during the study follow up period was recorded. ED visits separated by two days or less were counted as one episode (that is, only one ED visit) and ED visits followed by a hospital admission for asthma within 2 days of discharge were not counted. A minimum of 6 months of RAMQ drug plan coverage before and a minimum of 1 month after the ED visit were required to measure the use and predictors of use of ICS.

\section{Use of ICS after discharge}

Patients were considered to be users of ICS (beclomethasone, fluticasone, budesonide, flunisolide, triamcinolone) for a specific ED visit if they filled at least one prescription during the month following discharge or if they filled a prescription within 6 months before the ED visit that was still valid after discharge. A prescription was considered valid if the date of discharge from the ED was included in the time period estimated for its duration (estimated by the number of days between this refill and the previous one).

Using the same definition, we also assessed the use of ICS within the first 3 months and the first 6 months following ED discharge. These measures were taken to investigate whether ICS use improved over time.

\section{Statistical analyses}

The proportion of patients using ICS in the month following ED discharge was estimated as a function of patient characteristics such as sociodemographic factors, co-morbidity, severity of the exacerbation, prior use of ICS, and markers of asthma severity and control. It is worth noting that the unit of analysis was the ED visit and that a patient might have had more than one ED visit during the study period. A maximum of four ED visits (that is, the first four ED visits) per patient were included in all analyses.

We also looked further into the use of ICS after discharge from the ED to differentiate between patients who filled a prescription for ICS during the month following discharge and those who had only a valid prescription after discharge that was filled before the ED visit under study. We then estimated the proportion of patients using ICS in the first 3 and 6 months following a visit to an ED.

Following these descriptive analyses we investigated the predictors of ICS use in the month following discharge from the ED with a generalised estimation equation (GEE) model. Using this model we were able to include up to four ED visits per patient in the analysis and take into account the fact that a considerable proportion of patients had more than one ED visit during the study period. Mathematically, this model took into account the correlation between different ED visits for the same patient in the estimation of the confidence interval of the odds ratios (ORs) associated with the predictors under study. From this model we thus estimated adjusted ORs and 95\% CI for the use of ICS in the month following discharge from the ED for the following variables:

- socioeconomic factors: age at ED visit (13-17 v 512 years), sex (male $v$ female), living in a family receiving social assistance (yes/no), living in Montreal Island (yes/ no);

- co-morbidity: filling at least one prescription for a medication prescribed for a chronic condition other than asthma within 6 months before the ED visit (yes/no);

- severity of the exacerbation: consultation with a paediatrician or a respiratory physician during the ED visit (yes/ no); prescription filled for an oral corticosteroid in the month following ED discharge (yes/no);

- prior use of ICS: at least one prescription filled for an ICS (yes/no, excluding when relevant the prescription that was still valid at discharge) within 6 months before the ED visit;

- marker of asthma severity and control (measured within 6 months before the ED visit); at least one prescription filled for an oral corticosteroid (yes/no), average use of 10 or more doses of short acting $\beta_{2}$ agonists per week (yes/ no), at least one prescription filled for a long acting $\beta_{2}$ agonist (yes/no), hospital admission for asthma (yes/no), ED visit for asthma (yes/no), medical visit to a paediatrician or a respiratory physician (yes/no).

The use of short acting $\beta_{2}$ agonists as a marker of asthma control is based on the recommendation of the Canadian asthma guidelines which states that asthma is well controlled if a patient uses less than four doses per week, excluding one dose per day for exercise-that is, 10 doses per week or more. ${ }^{1}$

All analyses were performed on the first four ED visits of each patient. This maximum of four visits per patient was set in order to stabilise the GEE model. Among patients who had an ED visit during the study period, only $3.7 \%$ had more than four visits. The conclusions about the effect of the predictors under study on the likelihood of using ICS after an ED visit were only based on the results provided by the GEE model and not on the descriptive analyses which did not take into account the correlation between the different ED visits of a patient.

\section{RESULTS}

Of the 32897 asthmatic patients identified from the RAMQ database, 4042 met the inclusion criteria to enter the ED cohort. Of these, $68.2 \%$ had only one ED visit during the study period, $18.4 \%$ had two, $6.7 \%$ had three, and $6.7 \%$ had four or more.

The characteristics of the study patients are shown in table 1 . More than $70 \%$ of patients visiting the ED were aged $5-12$ years and $52 \%$ were male. In $24.3 \%$ of cases a 
Table 1 Characteristics of patients and use of ICS in the month following discharge from the ED

\begin{tabular}{|c|c|c|}
\hline & $\begin{array}{l}\text { No of visits } \\
(\%)\end{array}$ & $\begin{array}{l}\text { Use of ICS } \\
\text { (\%) }\end{array}$ \\
\hline All ED visits & $6143(100)$ & 63.0 \\
\hline \multirow{2}{*}{\multicolumn{3}{|c|}{$\begin{array}{l}\text { Sociodemographic factors } \\
\text { Age at ED visit (years) }\end{array}$}} \\
\hline & & \\
\hline $5-12$ & $4340(70.6)$ & 68.0 \\
\hline $13-17$ & $1803(29.4)$ & 50.9 \\
\hline \multicolumn{3}{|l|}{ Sex } \\
\hline Male & $3221(52.4)$ & 64.6 \\
\hline Female & $2922(47.6)$ & 61.2 \\
\hline \multicolumn{3}{|l|}{ Social assistance } \\
\hline Yes & $2996(48.8)$ & 62.1 \\
\hline No & 3147 (51.2) & 63.8 \\
\hline \multicolumn{3}{|l|}{ Living on Montreal Island } \\
\hline Yes & $1950(31.7)$ & 62.3 \\
\hline No & $4193(68.3)$ & 63.3 \\
\hline \multicolumn{3}{|l|}{ Co-morbidity* } \\
\hline Yes & $929(15.1)$ & 61.4 \\
\hline No & $5214(84.9)$ & 63.3 \\
\hline \multirow{2}{*}{\multicolumn{3}{|c|}{$\begin{array}{l}\text { Severity of the exacerbation } \\
\text { Consultation with a paediatrician or } \\
\text { respiratory physician }\end{array}$}} \\
\hline & & \\
\hline Yes & $1495(24.3)$ & 66.4 \\
\hline No & $4648(75.7)$ & 61.9 \\
\hline \multicolumn{3}{|c|}{ Prescription of oral corticosteroids } \\
\hline Yes & $2499(40.7)$ & 76.2 \\
\hline No & $3644(59.3)$ & 53.9 \\
\hline \multicolumn{3}{|c|}{ Use of ICS in 6 months before ED visit } \\
\hline Yes & $2373(38.6)$ & 79.4 \\
\hline No & $3770(61.3)$ & 52.6 \\
\hline \multirow{3}{*}{\multicolumn{3}{|c|}{$\begin{array}{l}\text { Markers of asthma severity and control } \\
6 \text { months before ED visit } \\
\text { Oral corticosteroids }\end{array}$}} \\
\hline & & \\
\hline & & \\
\hline Yes & $1471(23.9)$ & 76.9 \\
\hline No & $4672(76.1)$ & 58.6 \\
\hline \multicolumn{3}{|l|}{ Short acting $\beta_{2}$ agonists } \\
\hline$>10$ doses per week & 4641 (75.5) & 77.1 \\
\hline$\leqslant 10$ doses per week & $1502(24.5)$ & 58.4 \\
\hline \multicolumn{3}{|l|}{ Long acting $\beta_{2}$ agonists } \\
\hline Yes & $67(1.1)$ & 77.6 \\
\hline No & 6076 (98.9) & 62.8 \\
\hline \multicolumn{3}{|l|}{ Hospital admission for asthma } \\
\hline Yes & $337(5.5)$ & 76.3 \\
\hline No & 5806 (94.5) & 62.2 \\
\hline \multicolumn{3}{|l|}{ ED visit for asthma } \\
\hline Yes & $1643(26.7)$ & 67.9 \\
\hline No & 4500 (73.3) & 61.2 \\
\hline \multicolumn{3}{|c|}{$\begin{array}{l}\text { Medical visit to a paediatrician or a } \\
\text { respiratory physician }\end{array}$} \\
\hline $\begin{array}{l}\text { Yes } \\
\text { Yontion }\end{array}$ & $1861(30.3)$ & 77.9 \\
\hline No & $4282(69.7)$ & 56.5 \\
\hline
\end{tabular}

paediatrician or a respiratory physician was consulted during the ED visit and $40 \%$ of patients filled a prescription for oral corticosteroids in the month following discharge. This cohort is likely to represent patients with uncontrolled asthma since $76 \%$ of them used an average of 10 doses or more of short acting $\beta_{2}$ agonists per week, $24 \%$ filled at least one prescription for oral corticosteroids, $27 \%$ had an ED visit for asthma, and nearly $6 \%$ had a hospital admission for asthma during the 6 months preceding the ED visit.

Table 1 also shows the use of ICS in the month following ED discharge as a function of the characteristics of patients and physicians. Overall, patients filled a prescription of ICS in the month following discharge $63 \%$ of the time. Children were $17 \%$ more likely than adolescents to use ICS in the month following discharge. Patients who had at least one comorbidity were slightly less likely to use ICS after discharge than patients without co-morbidity. The most prevalent comorbidities observed in the cohort were psychiatric disorders $(3.8 \%)$, dermatological diseases $(3.4 \%)$, rhinitis $(3.0 \%)$, and musculoskeletal disorders $(3.0 \%)$. Patients who used asthma medications recognised as markers of uncontrolled asthma
Table 2 Use of ICS during 6 months after discharge from the ED (\%)

\begin{tabular}{lll}
\hline Use of ICS & $\begin{array}{l}5-12 \text { years } \\
(\mathbf{n}=\mathbf{4 3 4 0 )}\end{array}$ & $\begin{array}{l}\text { 13-17 years } \\
(\mathbf{n}=\mathbf{1 8 0 3})\end{array}$ \\
\hline Within 1 month & 68.0 & 50.9 \\
Within 3 months & 76.7 & 59.3 \\
Within 6 months & 77.2 & 60.1 \\
\hline
\end{tabular}

such as oral corticosteroids and high dose short acting $\beta_{2}$ agonists before the ED visit were more likely to use ICS after discharge. Patients who had been admitted to hospital during the 6 months before the ED visit were 14\% more likely to use ICS after discharge than those who were not admitted to hospital. Surprisingly, patients who had an ED visit in the 6 months before the ED visit under investigation were only $6.7 \%$ more likely to use ICS after discharge. We also observed that nearly $24 \%$ of patients who filled a prescription for oral corticosteroids in the month following discharge from the ED did not have a valid prescription for ICS during the same period. Finally, patients who had at least one medical visit to a paediatrician or a respiratory physician before the ED visit were $21 \%$ more likely to use ICS after discharge.

We looked further into ICS use during the first month following discharge from the ED among the 2951 children ( $68.0 \%$ of all children) classified as users of ICS; $66 \%$ of these children $(n=1948)$ had filled a prescription for ICS in the month following discharge from the ED while $34 \%$ $(n=1003)$ only had a prescription filled before the ED visit which was considered valid after discharge. The corresponding figures were $67 \%(n=615)$ and $33 \%(n=303)$ among adolescents.

The use of ICS in the 6 months after discharge from the ED is shown in table 2 . The proportion of children using ICS increased by $8.7 \%$ (from $68.0 \%$ to $76.7 \%$ ) between the first and third month and by only $0.5 \%$ (from $76.7 \%$ to $77.2 \%$ ) between the third and sixth month following ED discharge. The corresponding figures for adolescents were $8.4 \%$ and $0.8 \%$.

Crude and adjusted odds ratios were then estimated for all potential predictors of ICS use during the month after ED discharge (table 3 ). The two strongest predictors of ICS use were filling a prescription for oral corticosteroids in the month following discharge (adjusted OR 2.31 (95\% CI 2.05 to 2.60 )) and prior use of ICS (adjusted OR 2.35 (95\% CI 2.06 to $2.69)$ ). Patients who filled at least one prescription for oral corticosteroids (adjusted OR 1.45) used high doses $(>10$ doses/week) of short acting $\beta_{2}$ agonists (adjusted OR 1.57) and those who had a visit with a paediatrician or a respiratory physician during the 6 months before an ED visit (adjusted OR 1.67) were also significantly more likely to use ICS after ED discharge. We also observed that adolescents were less likely to use ICS after discharge than children (adjusted OR 0.50). On the other hand, consultation with a paediatrician or a respiratory physician during the ED visit and prior hospital admissions and ED visits for asthma were not significantly associated with the use of ICS after discharge. In fact, although non-significant, the adjusted ORs found in the model indicated that patients with prior ED visits or hospital admissions for asthma would be less likely to use ICS after an ED visit.

\section{DISCUSSION}

The results of this study show that nearly $50 \%$ of adolescents and $32 \%$ of children did not have a valid prescription for ICS during the month following discharge from the ED. These results are in agreement with those found in a study 
Table 3 Predictors of ICS use in the month following discharge from the ED

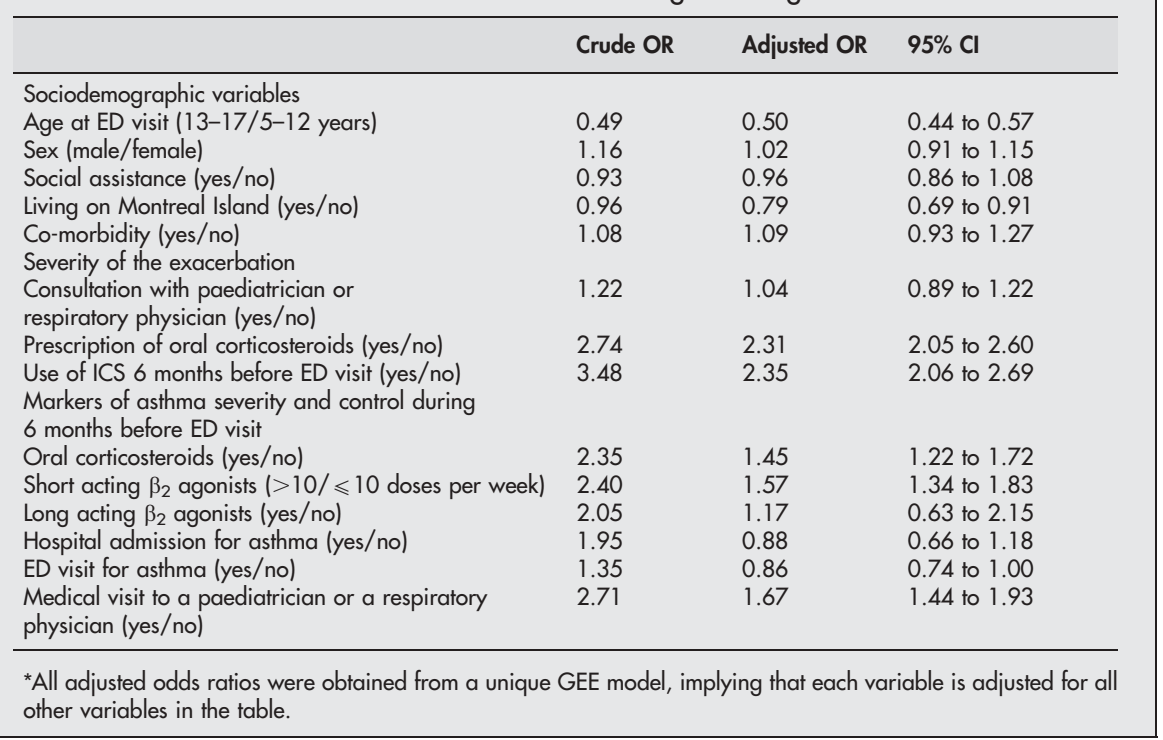

conducted in the Canadian province of Saskatchewan which found that $25 \%$ of adult patients were discharged from the ED without a prescription for ICS. ${ }^{8}$ Our results indicate, however, that the situation might be more problematic for adolescents than for adults and children since the proportion of patients without a valid prescription for ICS after discharge was much lower in this group of patients.

Our results also show that the use of ICS after a visit to the ED does not improve over time. In fact, patients were less likely to fill a prescription for ICS as time evolved after discharge from the ED for an asthma exacerbation. For both children and adolescents, only an additional $10 \%$ of patients filled a prescription for ICS between the second and sixth month following ED discharge. This might indicate that patients who were discharged from the ED and did not use ICS in the following month-either because they were discharged without a prescription or because they did not fill the prescription they received-have very little chance of getting one in the future from a family physician or changing their behaviour to these medications.

The stratification of patient age was based on an anticipated difference in behaviour related to the management of medications. The cut off at 13 years to define adolescence was thought to be related to the age at which patients are likely to start being responsible for the management of their own medications for asthma. As expected, the use of ICS by patients aged 13-17 years after discharge from the ED was quite different from that of younger patients who were probably closely supervised by their parents.

Analysis of the predictors of ICS use shows that patients with the most severe markers of uncontrolled asthma-that is, patients with prior ED visits and hospital admissions for asthma and those who had a consultation with a respiratory physician or a paediatrician during the ED visit-were not more likely to use ICS after discharge. In other words, patients who had two or more ED visits for asthma within a 6 month period were not more likely to use ICS after discharge than other patients. We also observed that $23 \%$ of patients who filled a prescription for oral corticosteroids in the month following discharge from the ED did not have any valid prescription for ICS during the same period. These results might reflect a vicious circle where uncontrolled patients are more likely to be admitted to the ED and less likely to use ICS, which in turn keeps the asthma uncontrolled and increases the likelihood of a severe exacerbation requiring acute care. This reasoning is further supported by the fact that we found that the use of ICS before the ED visit was one of the strongest predictors of ICS use after discharge.

Patients trapped in such a vicious circle might not have adequate outpatient care-including access to a centre for asthma education - to break the circle. Residents of Quebec are not required to be registered with a family physician and a significant proportion of patients do not have one. Visits to the ED are therefore a common means of obtaining health care. ED visits could then be seen as a good opportunity (or the only opportunity in certain cases) to teach and encourage patients-and parents for children-to take controller therapy to reduce the risk of a future asthma exacerbation requiring a visit to the ED. Patient education might be even more important for adolescents who may be more reluctant than other patients to fill the prescriptions received at discharge.

The results of this study are highly representative of real life practice for the treatment of asthma in Canadian institutions and other tertiary care institutions since they are based on a large sample of patients retrieved from an administrative database. Using the RAMQ database allowed us to avoid recall bias for drug consumption and changes in medical practice due to the participation of physicians in a research project. Moreover, we were able to follow patients for up to 3 years and include up to four ED visits per patient in the analyses.

Inevitably, the use of administrative databases for such a study has limitations. Firstly, the use of ICS was based on the filling of prescriptions which might not accurately reflect intake of the medication. The definition we used to identify ICS users (especially considering a patient to be a user even if his only valid prescription for ICS found after discharge was filled before the ED) is likely to overestimate the number of patients using these medications after discharge and to underestimate the number of patients using ICS before discharge. This misclassification of ICS exposure, if present, might have underestimated the impact of prior use of ICS and other factors on the likelihood of use of ICS after discharge. ${ }^{23}$ Secondly, the diagnosis of asthma for the ED visit recorded in the database was not validated. However, we feel confident that a large proportion of these patients have asthma since $76 \%$ of them used an average of more than 10 doses of short acting $\beta_{2}$ agonists per week before the ED visit. 
Thirdly, in this study it was not possible to differentiate between the behaviour of patients and physicians. It was not possible to know whether patients were classified as nonusers of ICS because they did not fill their prescriptions or because the ED physician did not prescribe them. Fourthly, our cohort is a representative sample of Quebec residents covered by the RAMQ drug plan, but might not be a good representation of families with the highest incomes. However, a priori, there is no reason to believe that patients will be treated differently at the ED based on their socioeconomic status. In our model receiving social assistance was not significantly associated with the use of ICS after discharge. Finally, we had no access to clinical markers of asthma severity and control such as lung function measures or the frequency of symptoms. The markers that we used were based on the use of asthma medications and acute care services for asthma before the ED visit. These markers were evaluated over a 6 month period that was chosen to be a trade off between the proximity of the ED visit under study and a good representation of the underlying severity and control. It is worth noting that our markers of control are more likely to represent the long term than an acute phase of lack of control since they were evaluated over a 6 month period.

In conclusion, despite clear recommendations from the Canadian guidelines for the treatment of asthma after an ED visit, we found that a large proportion of patients did not use ICS after discharge and this was even more important among adolescents. A large proportion of patients with clear markers of uncontrolled or severe asthma-such as prior ED visits and hospital admissions for asthma-did not have a valid prescription for an ICS after discharge. Prescription of an ICS and promoting patient compliance should be emphasised in children and adolescents with prior ED visits or hospital admissions for asthma.

\section{Authors' affiliations}

L Blais, M-F Beauchesne, Faculty of Pharmacy, Université de Montréal and Hôpital du Sacré-Coeur de Montréal, Endowment Chair Astra Zenecov in Respiratory Health, Montréal, Canada

Lucie Blais is the recipient of a New Investigator salary support grant from the Canadian Institutes for Health Research (ClHR). This study was supported by a grant from the Association Pulmonaire du Québec.

\section{REFERENCES}

1 Boulet L-P, Becker A, Beveridge R, et al. Canadian Asthma Consensus Report, 1999. Canadian Asthma Consensus Group. Can Med Assoc J 1999;161:S1-61.
2 Boulet L-P, Bérubé D, Cockcroft D, et al. Asthma guidelines update 2001. Can Respir J $2001 ; 8: 5-48$ A.

3 Murphy M, Bleecker ER, Ford JG, et al. Guidelines for the diagnosis and management of asthma. Clinical practice guidelines, Expert Panel Report 2.Publication no 97-4051. Bethesda, MD: NIH/NHLBI, 1997.

4 Rowe B, Bota G, Fabris L, et al. Inhaled budesonide in addition to oral corticosteroids to prevent asthma relapse following discharge from the emergency department: a randomized controlled trial. JAMA 1999;281:21 19-26.

5 Sin D, Man S. Low-dose inhaled corticosteroid therapy and risk of emergency department visits for asthma. Arch Intern Med 2002;162:1591-5.

6 Salmeron S, Liard R, Elkharrat D, et al. Asthma severity and adequacy of management in accident and emergency departments in France: a prospective study. Lancet 2001;358:629-35.

7 Robinson SM, Harrison BD, Lambert MA. Effect of a preprinted form on the management of acute asthma in an accident and emergency department. Emerg Med J 1996;13:93-7.

8 Stell IM. Asthma management in accident and emergency and the BTS guidelines: a study of the impact of clinical audit. Emerg Med $J$ 1996;13:392-4.

9 Scribano PV, Lerer T, Kennedy D, et al. Provider adherence to a clinical practice guideline for acute asthma in a pediatric emergency department. Acad Emerg Med 2001;8:1147-52.

10 Mahadevan M, Jin A, Manning P, et al. Emergency department asthma: compliance with an evidence-based management algorithm. Ann Acad Med Singapore 2002;31:419-24.

11 Crain EF, Mortimer KM, Bauman $\sqcup$, et al. Pediatric asthma care in the emergency department: measuring the quality of history-taking and discharge planning. J Asthma 1999;36:129-38.

12 Reid J, Marciniuk D, Cockcroft D. Asthma management in the emergency department. Can Respir J 2000;7:255-60.

13 Emerman CL, Cydulka RK, Skobeloff E. Survey of asthma practice among emergency physicians. Chest 1996;109:708-12.

14 Grunfeld A, Beveridge RC, Berkowitz J, et al. Management of acute asthma in Canada: an assessment of emergency physician behaviour. J Emerg Med 1997; 15:547-56

15 Singh A, Woodruff P, Ritz R, et al. Inhaled corticosteroids for asthma: are ED visits a missed opportunity for prevention? Am J Emerg Med 1999; 17:144-7.

16 Greenland S. Dose-response and trend analysis in epidemiology: alternatives to categorical analysis. Epidemiology 1995;6:356-65.

17 Blais L, Boucher JM, Couture J, et al. Impact of a cost-sharing drug insurance plan on drug utilization among older people. J Am Geriatr Soc $2001 ; 49: 410-4$.

18 Blais L, Desgagne A, LeLorier J. 3-Hydroxy-3-methylglutaryl coenzyme A reductase inhibitors and the risk of cancer: a nested case-control study. Arch Intern Med 2000;160:2363-8.

19 Blais R, Grégoire J-P, Rouleau R, et al. Ambulatory use of inhaled $\beta_{2}$-agonists for the treatment of asthma in Quebec. A population-based utilization review. Chest 2001;119:1316-21.

20 Garbe E, Lelorier J, Boivin J-F, et al. Inhaled and nasal glucocorticoids and the risks of ocular hypertension or open-angle glaucoma. JAMA 1997;277:722-7.

21 World Health Organisation. Guidelines for DDD. Oslo: WHO Collaborating Centre for Drug Statistics Methodology, 1993.

22 Tamblyn R, Lavoie G, Petrella L, et al. The use of prescription claims databases in pharmacoepidemiological research: the accuracy and comprehensiveness of the prescription claims database in Québec. J Clin Epidemiol 1995;48:999-1009.

23 Rothman KJ, Greenland S. Modern epidemiology, 2nd ed. Philadelphia: Lippincott-Raven Publishers, 1998. 